[0212-7199 (2005) 22: 9; pp 429-430] ANALES DE MEDICINA INTERNA Copyright $\odot 2005$ ARAN EDICIONES, S.L.

AN. MED INTERNA (Madrid) Vol. 22, N. ${ }^{\circ}$ 9, pp. 429-430, 2005

\title{
Fracaso hepático agudo tras golpe de calor
}

\author{
A. J. PARDO CABELLO, M. N. BENTICUAGA MARTÍNEZ, A.MARTÍN MORENO, \\ F. J. MIRAS PARRA, F. SABIO REYES ${ }^{1}$
}

Servicio de Medicina Interna B. ${ }^{1}$ Servicio de Urgencias y Emergencias. Hospital Clínico

San Cecilio. Granada

ACUTE LIVER FAILURE FOLLOWING HEAT STROKE

\begin{abstract}
RESUMEN
La afectación hepática en el curso de un golpe de calor suele ser un proceso frecuente, necesitando en algunos casos muy graves y raros de la realización de trasplante hepático. Presentamos el caso de un paciente varón de 31 años, deportista amateur, que mientras participaba en una competición de maratón sufrió un golpe de calor con fracaso hepático agudo acompañado de rabdomiolisis, insuficiencia renal y coagulopatía. La elevada temperatura ambiental, la duración del ejercicio y la altura a la que se realizó pudieron contribuir a la aparición del cuadro. La evolución del paciente, tras la aplicación de medidas conservadoras, fue favorable recibiendo el alta hospitalaria en pocos días.
\end{abstract}

PALABRAS CLAVE: Golpe de calor. Fracaso hepático agudo. Rabdomiolisis. Fracaso multiorgánico.

\begin{abstract}
Hepatic involvement during heat stroke appears frequently. In some severe and rare cases liver transplantation is needed. We report a case of a 31 years old man, amateur runner, who suffered heat stroke-related acute liver failure, rhabdomyolysis, renal failure and coagulation impairment during a marathon. High environmental temperature, exercise duration and height where race took place could be involved. Patient had a favourable course with conservative treatment being discharged in a few days.
\end{abstract}

KEY WORDS: Heat stroke. Acute liver failure. Rhabdomyolysis. Multiple organ failure.

Pardo Cabello AJ, Benticuaga Martínez MN, Martín Moreno A, Miras Parra FJ, Sabio Reyes F. Fracaso hepático agudo tras golpe de calor. An Med Interna (Madrid) 2005; 22: 429-430.

\section{INTRODUCCIÓN}

El golpe de calor tras ejercicio aparece en situaciones en las que se genera por ejercicio muscular un calor superior al que el organismo es capaz de disipar (1). Los pacientes afectados por esta patología suelen presentar una alteración de sus parámetros hepáticos, renales y musculares generalmente secundaria a la isquemia tisular que se produce en el contexto de la hipoperfusión que afecta al organismo.

\section{CASO APORTADO}

Se trata de un varón de 31 años de edad, deportista aficionado, sin antecedentes de interés ni consumo previo de tóxicos, que tras un intenso ejercicio físico al sol (4 horas de maratón en Sierra Nevada) presentó una pérdida de conocimiento con caída al suelo metros antes de su llegada a meta acompañados de vómitos alimenticios. Es atendido por Servicio de Urgencias extrahospitalarias que evidencia taquicardia y una temperatura de $38,5^{\circ} \mathrm{C}$. Desde allí avisan al 061 que lo encuentra estable hemodinámicamente con hiperventilación y agitación por lo que se administra midazolam IV trasladandose posteriormente al Servicio de Urgencias de nuestro Hospital. En el área de Observación se objetiva una temperatura de $40^{\circ} \mathrm{C}$ que precisa de medios físicos, paracetamol y metimazol IV a lo largo de 3 horas para su corrección y un síndrome confusional que mejora paulatinamente con estabilidad hemodinámica y buena función respiratoria. En la analítica realizada en Urgencias destaca una elevación de CPK y LDH junto a un discreto aumento de transaminasas, urea y creatinina (Tabla I). Tras permanecer 24 horas en Observación pasa al S. Medicina Intensiva donde ingresa consciente y orientado, bien hidratado y perfundido, eupneico y con estabilidad hemodinámica. La temperatura en ese momento es de $37^{\circ} \mathrm{C}$ y el resto de las constantes vitales normales. La exploración cardiorrespiratoria, abdominal y de extremidades era normal. El ECG en ese momento refleja un ritmo sinusal a $80 \mathrm{lpm}$, PR normal con BRDHH incompleto sin signos de isquemia ni alteraciones de la repolarización.

Durante la estancia en UCI el paciente mantiene la consciencia en todo momento sin signos de encefalopatía y con dolores musculares de media intensidad como única sintomatología. En la UCI, en el segundo día desde ingreso se evidencia disfunción multiorgánica con afectación renal, fallo hepático agudo (llegándose a 8.770 U/l de GPT y 8.440 de GOT) con coagulopatía secundaria (protrombina $24 \%$ y TPTA 48 "), plaquetopenia por consumo (37.000) y rabdomiolisis (con mioglobina

Trabajo aceptado: 20 de abril de 2005 
TABLA I

RESULTADOS DE ANALÍTICA SANGUÍNEA DURANTE EL INGRESO

\begin{tabular}{lccccc}
\hline & Día 1 & Día 2 & Día 5 & Día 6 & $\begin{array}{c}\text { Día 12. } \\
\text { Alta }\end{array}$ \\
\hline CPK(U/l) & 1076 & 70668 & 26242 & 21550 & 395 \\
LDH(U/l) & 860 & 14023 & 2112 & 1561 & 481 \\
Mioglobina(U/l) & $\cdots-.-$ & $>4000$ & $>4000$ & 2596 & 140 \\
COT(U/l) & 54 & 8440 & 1150 & 823 & 79 \\
GPT(U/l) & 28 & 8770 & 3350 & 1245 & 377 \\
Urea(mg/dl) & 58 & 53 & 24 & 34 & 25 \\
Creatinina (mg/dl) & 2,2 & 1,4 & 1,1 & 1,3 & 0.9 \\
Plaquetas//mm³) & 268.000 & 37.000 & 118.000 & 155.000 & 405000 \\
Actividad protrombina (\%) & 66 & 24 & 73 & 97 & 92 \\
TPTA(seg) & 3 & 48 & 42 & 38 & 40 \\
\hline
\end{tabular}

mayor de $4000 \mathrm{U} / \mathrm{l})$. Se le administró manitol 10\%, furosemida, suero glucosalino y salino; suero bicarbonatado hasta alcalinización de orina para evitar precipitación de mioglobina; gastroprotección; vitamina $\mathrm{K}$ $\mathrm{IV}, 2.000 \mathrm{ml}$ de plasma fresco y 10 Unidades de plaquetas para restaurar sistemas de coagulación y recuento plaquetar.

$\mathrm{Al}$ quinto día, destacaba ictericia marcada a nivel conjuntival y diuresis de $8.000 \mathrm{ml} / 24 \mathrm{~h}$. La analítica reflejaba una elevación de bilirrubinas directa $(3,2)$ e indirecta $(3,9)$ además de otros parámetros (ver tabla 1). Pasa al Servicio de Medicina Interna para controlar su evolución y tratamiento. Al ingreso en Medicina Interna destaca un cuadro febril de $38^{\circ} \mathrm{C}$, cuyo origen era urinario, que se trata con antibioterapia convencional desapareciendo la fiebre y las molestias. Se continua diuresis alcalina forzada durante 2 dias y se realizan controles analíticos seriados hasta la normalización de los valores. En el momento del alta hospitalaria, tras otros siete días de estancia en este Servicio, el paciente presenta una urea de 25; creatinina 0,9; GOT 79; GPT 377; BD 0,9 у BI 1,1; LDH 481; CPK 395; TPTA 40"; 405000 plaquetas; mioglobina 140 y troponina 0,03. Clínicamente se encontraba asintomático.

\section{DISCUSIÓN}

En este paciente, como en la mayoría de los afectados por un golpe de calor, se pudo objetivar secuencialmente, tras la realización de un ejercicio físico extenuante, bajo unas condiciones de calor ambiental y baja presión de oxígeno (día soleado y en altura) importantes, una sucesión de síntomas y signos que orientan al diagnóstico como son: pérdida de consciencia, situación de hipertermia y alteraciones derivadas de la isquemia sistémica.
El golpe de calor se puede definir como una grave situación caracterizada por una temperatura central mayor de $40^{\circ} \mathrm{C}$ con anormalidades del SNC como delirium, convulsiones o coma que resultan de una exposición al calor ambiental (golpe de calor clásico) o ejercicio físico extenuante (golpe de calor del ejercicio).

En el golpe de calor generalmente se produce un shock circulatorio secundario a un fallo en la termorregulación. La respuesta termorreguladora normal se encamina a producir una vasodilatación cutánea y una vasoconstricción esplácnica que cuando se mantienen dan lugar a la hiperproducción de mediadores químicos (NO, radicales libres...) que generan un fallo circulatorio. Una vez se produce el fallo circulatorio se desencadena la hipoperfusión tisular que se refleja en la elevación de marcadores de sufrimiento de órganos como hígado, riñón... y a veces de coagulopatía que puede llegar a CID (2).

Hace años se informó de los cambios producidos en los hígados de ratas sometidas a sobrecarga de calor que se asemejan a los producidos en humanos: necrosis lobulillar, vacuolización y disminución de microvellosidades hepatocelulares y la pérdida del endotelio sinusoidal (3).

Posteriormente se han descrito casos de fallo multiorgánico por golpe de calor similar al de nuestro paciente: un paciente presentó un golpe de calor con fallo renal (urea 96 y creatinina 10), rabdomiolisis $($ CPK > 1000) y fallo hepático con GPT > 650 y protrombina 51" (4). Dicho paciente permaneció un mes inconsciente y después se recuperó mostrando una normalización de función renal a las 6 semanas tras tratamiento conservador.

En relación a las hepatitis hipóxicas producidas en estos pacientes debemos decir que se trata de una afectación relativamente frecuente. Sin embargo el fallo hepático es una situación rara que se asocia a un mal pronóstico $(1,4)$. El interés de nuestro caso radica en que se produjo una importante necrosis hepática isquémica alcanzándose cifras de transaminasas mayores de 8400 , TPTA de 48 segundos y protrombina de $24 \%$ que revirtieron tras tratamiento conservador. Tal cifra se alcanzó al segundo día tras el proceso coincidiendo con lo descrito en la literatura previa $(1,6,7)$.Son varios los autores que, como en nuestro caso, han obtenido un buen resultado con tratamiento conservador en esta situación $(1,3,6,7)$. En otros casos se han realizado, con muy pobres resultados, trasplantes urgentes de hígado (8-10).

Una buena estrategia para evitar golpes de calor sería disminuir los factores de riesgo a la hora de realizar un ejercicio físico o exponerse a un elevado calor ambiental como son limitar el tiempo de exposición, la ingesta de medicamentos (anticolinérgicos, neurolépticos...), realizar una adecuada hidratación durante el ejercicio y si no se puede evitar, intentar su diagnóstico y tratamiento precoces antes de que la situación llegue a ser irreversible.

\section{Bibliografía}

1. Hadad E, Ben-Ari Z, Heled Y, Moran D, Shani Y et al. Liver transplantation in exertional heat stroke: a medical dilemma. Intensive Care Med 2004; 30: 1474-8.

2. Bouchama A, Knochel JP. Heat Stroke. N Engl J Med 2002; 346:1978-88.

3. Bowers WD, Hubbard RW, Leav I, Daum R, Conlon M et al. Alterations of rat liver subsequent to heat overload. Arch Pathol Lab Med 1978; 102: 154-7.

4. Lumlertgul D, Chuaychoo B, Thitiarchakul S, Srimahachota S, Sangchun K et al. Ren Fail 1992; 14: 77-80.

5. Giercksky T, Boberg KM, Farstad IN, Halvorsen S, Schrumpf E. Severe liver failure in exertional heat stroke. Scand J Gastroenterol 1999; 34: 824-7.

6. Pastor MA, Perez-Aguilar F, Ortiz V, Nicolas D, Berenguer J. Acute hepatitis due to heat stroke. Gastroenterol Hepatol 1999; 22: 398-9.

7. Wagner M, Kaufmann P, Fickert P, Trauner M, Lackner C. Successful conservative management of acute hepatic failure following exertional heatstroke. Eur J Gastroenterol Hepatol 2003; 15: 1135-9.

8. Saissy JM, Almanza L, Samuel D, Pats B. Liver transplantation after exertioninduced heat stroke associated with fulminant liver failure. Presse Med 1996; 25: $977-9$.

9. Hassanein T, Perper JA, Tepperman L, Starlz TE, Van Thiel DH. Liver failure ocurring as a component of exertional heatstroke. Gastroenterology 1991; 100: 1442-7.

10. Berger J, Hart J, Millis M, Baker AL. Fulminant hepatic failure from heat stroke requiring liver transplantation. J Clin Gastroenterol 2000; 30: 429-31. 